\title{
介质阻挡放电微型化-长光程原子吸收光谱测定录及甲基录
}

\author{
于永亮 ${ }^{a}$ 高飞 ${ }^{b}$ 陈明丽 $b$ 王建华*, $b$ \\ $\left({ }^{a}\right.$ 东北大学化学系 沈阳 110819) \\ $\left({ }^{b}\right.$ 东北大学分析科学研究中心 沈阳 110819)
}

\begin{abstract}
摘要 以介质阻挡放电(DBD)为低温原子化器并引入长光程吸收检测池, 建立了微型化原子吸收光谱系统. 在顺序注 射系统中产生的录及甲基永蒸气依次经过气液分离器、玻璃棉除水微柱和原子化器然后进入长光程吸收检测池, 进行 原子吸收光谱测定. 当 DBD 原子化器关闭时, 通过冷原子吸收测得无机录的吸光度, 而当 DBD 原子化器开启时, 得到 无机录和甲基录的总吸光度. 在本体系中两种录形态的吸光度具有很好的加合性，从而有利于实现无机录和甲基录的 分别测定. 当进样体积为 $1.0 \mathrm{~mL}$ 时, 无机录与甲基录的检出限分别为 0.3 和 $0.4 \mu \mathrm{g} \cdot \mathrm{L}^{-1}$, 相对标准偏差均小于 $4 \%$. 用本 微型化原子吸收光谱系统测定了实际样品中的录及其形态, 证明了该系统的可靠性.
\end{abstract}

关键词介质阻挡放电；长光程; 原子吸收光谱; 录; 形态分析

\section{A Miniaturized Long-Optical Path Atomic Absorption Spectrometer with Dielectric Barrier Discharge as Atomizer for Mercury and Methylmercury}

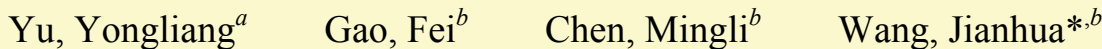 \\ ( ${ }^{a}$ Department of Chemistry, Northeastern University, Shenyang 110819) \\ ( ${ }^{b}$ Research Center for Analytical Sciences, Northeastern University, Shenyang 110819)
}

\begin{abstract}
The development of miniaturized instrumentations for specific analytical purposes has been one of the most important momentum in the field of instrumental innovation. Sequential injection system as a miniaturized analytical platform provides an alternative for field of analysis of heavy metal contaminants. It will exhibit more powerful capability with the introduction of atomization function and improvement of detection sensitivity. In this work, a miniature long-optical path atomic absorption spectrometric system is developed with dielectric barrier discharge (DBD) low temperature micro-plasma as atomizer. Mercury and methylmercury vapor is generated in a sequential injection system. It is directed to flow through a gas-liquid separator, a glass wool moisture-removal microcolumn and the DBD atomizer, and finally transported into the long optical-path detection cell for quantitative analysis by atomic absorption spectrometry. A $10 \mathrm{~mA}$ lamp current is used with a mercury hollow cathode lamp, and a $260 \mathrm{~V}$ negative high voltage for the photomultiplier is set. The detection sensitivity of the present system is highly improved by the increase of absorption optical path $(1.1 \mathrm{~mm}$ i.d., $400 \mathrm{~mm}$ length), and the atomization of hydride is completely achieved by the DBD micro-plasma atomizer. Meanwhile, a glass wool moisture-removal microcolumn integrated in the flow system effectively eliminates the influence of concomitant moisture during the vapor generation process, which avoids the drift of absorbance baseline. The absorbance arising from mercury is recorded by turning off the DBD atomizer, while the total absorbance from both mercury and methylmercury is measured by turning on the DBD atomizer. Further experiments demonstrated satisfactory additivity for the absorbance arising from inorganic and methyl mercury, which provides basis for the determination of mercury and methylmercury. With a sampling volume of 1.0 $\mathrm{mL}$, detection limits of 0.3 and $0.4 \mu \mathrm{g} \cdot \mathrm{L}^{-1}$ are achieved respectively for inorganic and methylmercury, along with RSD values of $<4 \%$. The reliability of the present system is demonstrated by analyzing certified reference materials and real samples for mercury speciation.
\end{abstract}

Keywords dielectric barrier discharge; long optical-path; atomic absorption spectrometry; mercury; speciation

\section{1 引言}

由于现代工业的排放和泄露, 经常造成突发的环境 污染事件，其中以含有毒重金属/非金属污染物为代表
的环境污染事故对公众健康具有极大的危害. 对于重金 属的现场分析测定，目前最为有效的分析手段和技术首 推原子光谱 ${ }^{[1,2]}$. 由于现场分析的特殊性，对分析技术和 相关仪器提出了更高的要求，从而使现场分析仪器的微

*E-mail: jianhuajrz@mail.neu.edu.cn; Tel.: 024-83688944; Fax: 024-83676698

Received April 18, 2013; published May 16, 2013.

Supporting information for this article is available free of charge via the Internet at http://sioc-journal.cn.

Project supported by the National Natural Science Foundation of China (Nos. 21005013, 21005011), the National Science \& Technology Pillar Program (No. 2012BAF14B09) and the Fundamental Research Funds for the Central Universities (Nos. N120505004, N110805001, N110705002).

项目受国家自然科学基金(Nos. 21005013，21005011)、国家科技支撑计划(No. 2012BAF14B09)和中央高校基本科研业务费(Nos. N120505004, N110805001, N110705002)资助. 
型化和便携化成了重要发展趋势 ${ }^{[3]}$. 微顺序注射分析系 统作为一种微型化分析操作平台, 可与光谱或电化学检 测单元组成联用系统, 并独立完成多种分析任务 ${ }^{[4]}$. Erxleben 等 ${ }^{[5]}$ 基于微顺序注射系统建立了一种检测痕量 采的微型化原子吸收光谱装置, 但该装置的测定灵敏度 较低(测定录的检出限仅为 $9 \mu \mathrm{g} \cdot \mathrm{L}^{-1}$ ), 而且由于不具备 原子化的功能, 使得该装置的实际应用能力受到很大限 制. 因此, 引入原子化器并提高检测灵敏度是微型原子 吸收光谱亟待解决的瓶颈问题. 介质阻挡放电(DBD)是 一种低温等离子体发生技术 ${ }^{[6,7]}$, 其所产生的自由基或 离子的化学性质非常活跃, 可有效地促进化学分子的分 解. 因此, DBD 可以作为挥发性组分的原子化器 ${ }^{[8,9]}$. 另 外, 辅之以长光程吸收检测(流通)池作为光谱吸收的检 测单元, 也可以显著地提高吸收光谱的检测灵敏 度 $^{[10 ~ 12]}$, 并可通过控制调节吸收池(或检测光路)的长度 而实现对灵敏度的任意调控, 这对于提高微型化原子吸 收光谱系统现场分析操作的可控性具有重要意义.

本文报道了一种微型化原子吸收光谱系统, 通过引 入 DBD 低温原子化器 ${ }^{[13]}$, 并集成长光程吸收检测池, 可有效地实现无机采、甲基录以及总采的分析测定. 由 于该微型化系统的分析性能较原有报道的系统大为提 高, 且具有便携性使其适合于突发性环境应急事件中重 金属丞的现场分析.

\section{2 结果与讨论}

\section{1 流通池的吸收光程}

在原子吸收光谱检测中, 测定的灵敏度很大程度上 取决于吸收光程. 当气相目标组分引入长光程吸收检测 池时, 在浓度不变的情况下, 在一定范围内增加吸收检 测池的吸收光程可以有效提高测定的灵敏度. 然而当吸 收池过长时, 入射光在吸收池中传输所产生的光强度耗 散增大可能会限制灵敏度提高. 在本实验中, 我们在一 定范围内考察了吸收检测池或光程的长度对录原子吸 收光谱信号强度的影响. 实验结果表明, 在 $50 \sim 400$ $\mathrm{mm}$ 范围内, 增加吸收检测池的长度可线性增大无机采 与甲基录的吸光度信号. 本研究中所采用的光源具有足 够的激发能量, 因此继续增加吸收池的光程(超过 400 $\mathrm{mm}$ ) 时, 依然可以提供足够的特征光谱辐射而进一步提 高检测的灵敏度. 考虑到吸收检测池是内径为 $1.1 \mathrm{~mm}$ 的石英管, 其长度太大时操作不方便, 而且当光程达到 $400 \mathrm{~mm}$ 时测定的灵敏度已可满足实际现场分析的需要, 故本研究中不再进一步增加吸收检测池的吸收光程, 而 最终确定为 $400 \mathrm{~mm}$, 同时选择光源的灯电流为 $10 \mathrm{~mA}$, 检测器的负高压为 $260 \mathrm{~V}$. 所设计的微型化原子吸收光 谱系统的实验流路如图 1 所示.

\section{2 伴生水蒸汽对测定的影响}

当利用丞蒸气发生向吸收检测池中引入样品时, 少

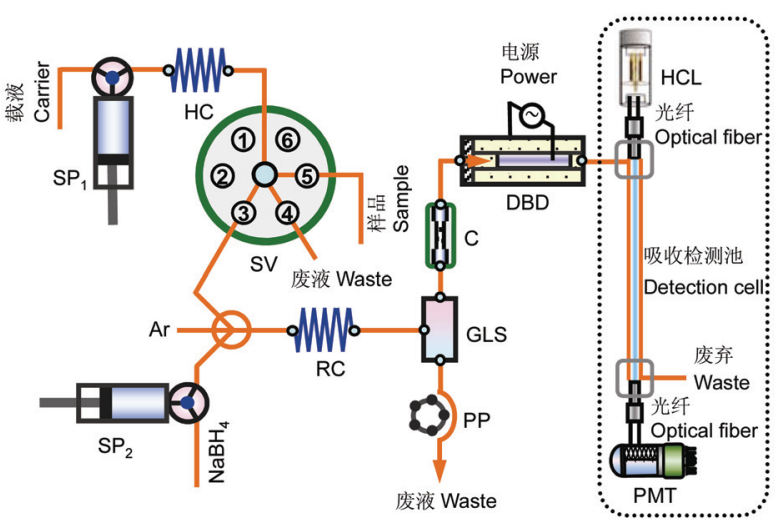

图 1 DBD 微型化超长光程原子吸收光谱系统及其流路示意图

Figure 1 Schematic diagram of the miniaturized long-optical path atomic absorption spectrometer with dielectric barrier discharge (DBD) as atomizer

SP1，SP2: 注射原(syringe pumps); SV: 多位选择阀(multi-port selection valve); PP: 蠕动原(peristaltic pump); HC: 储液环(holding coil); RC: 反应 环(reaction coil); GLS: 气液分离器(gas-liquid separator); C: 除水微柱 (moisture-removal microcolumn); DBD: 介质阻挡放电 (dielectric barrier discharge); HCL: 空心阴极灯 (hollow cathode lamp); PMT: 光电倍增管 (photomultiplier)

量的伴生水蒸汽也会随着永蒸气被一同引入吸收检测 池内. 由于水蒸汽分子对录特征谱线的较强吸收而严重 影响对录吸光度的测定, 所记录的信号峰形如图 2 所示. 当有水蒸汽伴随进入吸收检测池时, 其对光源特征谱线 的吸收致使检测器接收到的光强度下降, 从而使得吸光 度值变大，并导致基线发生严重漂移而干扰测定(图 2A). 为了防止少量伴生水蒸汽进入吸收检测池, 我们 在气液分离器和介质阻挡放电之间引入了一个除水微 柱，该微柱中填充适量玻璃棉用以吸收蒸气发生时所伴 生的少量水蒸汽. 实验结果表明这种方法可以有效地消 除水蒸汽对录原子吸收光谱测定的干扰，吸光度测定前 后基线没有漂移而基本保持在同一水平(图 2B). 值得一 提的是除水微柱中所用的玻璃棉无需经常更换，装填一 次可完成 200 次以上的分析测定.

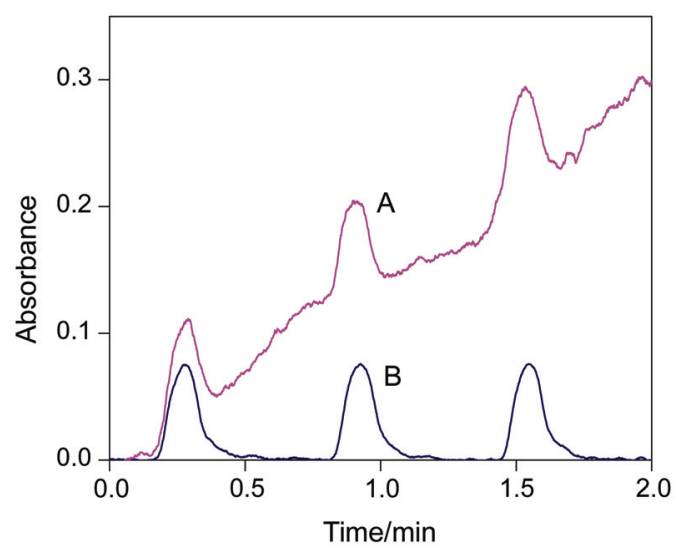

图 2 伴生水蒸汽对录原子吸收光谱信号的影响

Figure 2 The effect of moisture on the AAS signal profiles of mercury A: The signal for $5 \mu \mathrm{g}^{\circ} \mathrm{L}^{-1} \mathrm{Hg}^{2+}$ in the presence of concomitant moisture, B: The signal for $5 \mu \mathrm{g} \cdot \mathrm{L}^{-1} \mathrm{Hg}^{2+}$ after moisture removal 


\section{3 介质阻挡放电原子化与录形态分析}

当采用蒸气发生技术引入样品时, 无机丞可转化为 采原子蒸气而可直接进行冷原子吸收光谱测定; 然而, 在蒸气发生过程中甲基录转化为 $\mathrm{CH}_{3} \mathrm{HgH}$ 氢化物, 无法 用冷原子吸收光谱直接进行测定. 为了实现甲基录的原 子吸收光谱测定, 需要引入有效的原子化单元. 本实验 中采用介质阻挡放电作为低温原子化器, 当输入电压为 $50 \mathrm{~V}$ 时, 可在 DBD 中产生稳定的低温等离子体, 并实 现 $\mathrm{CH}_{3} \mathrm{HgH}$ 氢化物的原子化, 如图 3 所示. 在实际测定 中, 当 DBD 原子化器关闭时, 只获得无机录的吸收信号 峰, 甲基录的存在对无机采的信号峰没有明显影响; 当 DBD 原子化器开启时, 可同时获得无机丞和甲基录的 吸收信号峰, 而且 DBD 等离子体对无机采的信号峰没 有明显影响. 研究表明, 无机永和甲基录的吸光度具有 很好的加合性. 当样品中有无机采与甲基录共存时, 可 在先关闭 DBD 原子化器的条件下测得无机录的吸光度, 然后开启 $\mathrm{DBD}$ 原子化器测得无机永和甲基录的总吸光 度. 由于本系统中无机永与甲基永的吸光度具有很好的 可加和性, 可通过差减法同时得到无机录和甲基录的吸 光度, 从而实现样品中录的形态分析.

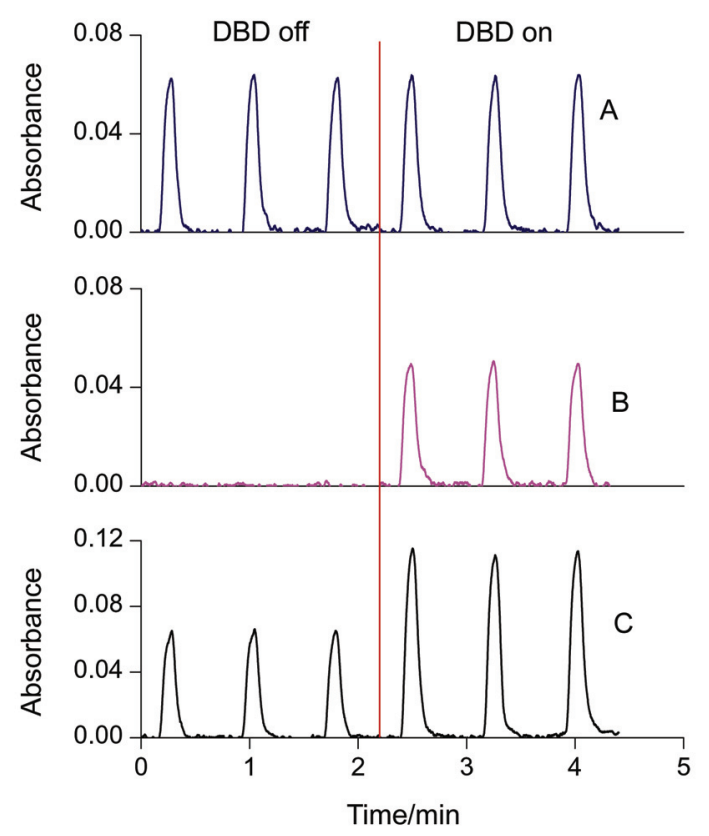

图 3 在 DBD 微型化超长光程原子吸收光谱系统中无机录与甲基录 的吸光度及其加合性

Figure 3 Recorded AAS signal (absorbance) for inorganic mercury and methylmercury in the long-optical path miniaturized DBD-AAS system A: $5 \mu \mathrm{g}^{\circ} \mathrm{L}^{-1} \mathrm{Hg}^{2+}$; B: $5 \mu \mathrm{g} \cdot \mathrm{L}^{-1} \mathrm{CH}_{3} \mathrm{Hg}^{+}$; $\mathrm{C}: 5 \mu \mathrm{g}^{\circ} \mathrm{L}^{-1} \mathrm{Hg}^{2+}+5 \mu \mathrm{g} \cdot \mathrm{L}^{-1} \mathrm{CH}_{3} \mathrm{Hg}^{+}$. $\mathrm{HCl}$ concentration: $0.5 \%(\phi) ; \mathrm{NaBH}_{4}$ concentration: $0.1 \mathrm{~g} \cdot \mathrm{L}^{-1}$; Argon flow rate: $150 \mathrm{~mL} \cdot \mathrm{min}^{-1}$.

\section{4 样品引入条件与干扰实验}

实验发现，当样品溶液的酸度处在 $0.2 \% \sim 3 \%$ $\mathrm{HCl}$ (浓盐酸体积和总体积比)范围内时, 所得到的无机
采和甲基录的吸光度信号基本保持不变，本实验中选择 $0.5 \% \mathrm{HCl}$ (浓盐酸体积和总体积比). 在此条件下当还原 剂 $\mathrm{NaBH}_{4}$ 浓度超过 $0.1 \mathrm{~g} \cdot \mathrm{L}^{-1}$ 时，即可同时实现无机录和 甲基录的蒸气发生，后续实验中选择 $\mathrm{NaBH}_{4}$ 的浓度为 $0.1 \mathrm{~g} \cdot \mathrm{L}^{-1}$. 另外, 较低的载气流速有利于避免对载气流 中无机采和甲基永蒸气浓度的稀释, 并可增加其在吸收 检测池中的停留时间，从而提高吸光度信号值; 另一方 面, 过低的载气流速也会导致吸光度信号峰变宽且稳定 性变差. 通过对载气流速在 $100 \sim 400 \mathrm{~mL} \cdot \mathrm{min}^{-1}$ 范围内 的考察发现 $150 \mathrm{~mL} \cdot \mathrm{min}^{-1}$ 的载气流速可获得满意的吸 光度信号.

我们考察了共存离子对录测定的干扰情况. 实验结 果表明, 利用此微型化长光程原子吸收光谱系统测定无 机录时，体系中共存 $5 \mathrm{mg} \cdot \mathrm{L}^{-1}$ 的 $\mathrm{Al}^{3+}, \mathrm{Zn}^{2+}, \mathrm{Fe}^{3+}, \mathrm{Cu}^{2+}$, $\mathrm{Ni}^{2+}, \mathrm{Cd}^{2+}, \mathrm{Cr}^{3+}, \mathrm{Pb}^{2+}, \mathrm{Co}^{2+}, \mathrm{AsO}_{2}^{-}$等离子对吸光度信 号的影响可有效地控制在 $\pm 5 \%$ 以内. 当同时测定无机 采和甲基录时，可允许的共存离子浓度相同，只是此时 对 $\mathrm{Cu}^{2+}$ 的允许浓度降低为 $0.5 \mathrm{mg} \cdot \mathrm{L}^{-1}$. 在实际样品测定 时，这些离子的浓度一般会被有效地控制在允许范围 内，因此对灵测定的影响可不予考虑.

\section{5 系统的分析性能}

在上述优化的实验条件下, 微型化原子吸收光谱系 统用于无机录和甲基录测定的分析性能概括如下：当进 样量为 $1.0 \mathrm{~mL}$ 时, 采样频率为 $78 \mathrm{~h}^{-1}$, 检出限分别为 0.3 和 $0.4 \mu \mathrm{g} \cdot \mathrm{L}^{-1}$ (空白测定标准偏差的 3 倍), 线性范围分别 为 $1.0 \sim 50$ 和 $1.2 \sim 50 \mu \mathrm{g} \cdot \mathrm{L}^{-1}$. 对 $2 \mu \mathrm{g} \cdot \mathrm{L}^{-1}$ 的无机录和甲 基录进行 7 次平行测定, 得到系统的精密度 RSD 分别为 $2.6 \%$ 和 $3.9 \%$.

\section{6 样品的分析应用}

为检验所建立的 DBD 微型化长光程原子吸收光谱 系统的可靠性, 我们分析了标准样品 GBW 09101(人 发)、CRM 176(城市垃圾焚烧灰烬)、金枪鱼肉和水样的 采, 并对金枪鱼肉和水样进行了加标回收实验, 结果如 表 1 所示. 很显然, 标准物质 GBW 09101 和 CRM 176 中采含量的测定值与标准值较吻合. 金枪鱼肉中未发现 游离的 $\mathrm{Hg}^{2+}$, 但测得一定浓度的甲基录，而湖水中未检 测出无机湬和甲基永. 另外, 对金枪鱼肉和湖水样品进 行无机采和甲基录的加标回收实验得到了满意的回收 率. 上述有关实验均证明了本系统的可行性.

\section{3 结论}

利用微顺序注射分析操作平台, 我们建立了一种微 型化原子吸收光谱系统. 通过采用长光程流通池作为光 谱吸收的检测单元，可显著提高吸收光谱的检测灵敏 度, 同时集成 DBD 低温原子化器, 可有效地实现无机 采、甲基录以及总丞的分析测定. 另外，引入玻璃棉微 
表 1 测定实际样品中的无机录和甲基录的含量 $(n=3)$

Table 1 Determination of inorganic mercury and methylmercury in certified reference materials and real samples $(n=3)$

\begin{tabular}{|c|c|c|c|c|c|c|c|}
\hline \multirow{2}{*}{ 被测物质 } & \multirow{2}{*}{ 总 $\mathrm{Hg}$ 标准值 $/\left(\mu \mathrm{g} \bullet \mathrm{g}^{-1}\right)$} & \multicolumn{2}{|c|}{ 测定值/( $\left.\mu \mathrm{g} \bullet \mathrm{g}^{-1}\right)$} & \multicolumn{2}{|c|}{ 加标 $/\left(\mu \mathrm{g} \cdot \mathrm{L}^{-1}\right)$} & \multicolumn{2}{|c|}{ 回收率/\% } \\
\hline & & $\mathrm{Hg}^{2+}$ & $\mathrm{CH}_{3} \mathrm{Hg}^{+}$ & $\mathrm{Hg}^{2+}$ & $\mathrm{CH}_{3} \mathrm{Hg}^{+}$ & $\mathrm{Hg}^{2+}$ & $\mathrm{CH}_{3} \mathrm{Hg}^{+}$ \\
\hline GBW 09101 & $2.16 \pm 0.21$ & $2.1 \pm 0.2$ & - & - & - & - & - \\
\hline CRM 176 & $31.4 \pm 1.1$ & $31 \pm 2$ & - & - & - & - & - \\
\hline \multirow[t]{2}{*}{ 金枪鱼肉 } & - & N.D..$^{a}$ & $0.24 \pm 0.01$ & - & $0.17^{b}$ & - & 95 \\
\hline & & & & 3 & 10 & 107 & 99 \\
\hline \multirow[t]{2}{*}{ 湖水 } & - & N.D. & N.D. & 5 & 5 & 102 & 99 \\
\hline & & & & 10 & 3 & 99 & 98 \\
\hline
\end{tabular}

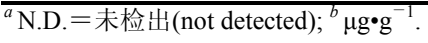

填充柱有效地消除了蒸气发生时所伴生的水蒸汽对永 吸光度测定的影响及其导致的基线漂移问题. 该微型化 系统的高灵敏度及其便携性使其适合于突发性环境应 急事件中重金属永的现场分析.

\section{References}

[1] Yu, Y.-L.; Wang, J.-H. Chin. Sci. Bull. 2013, doi: 10. 1007/s11434-013-5666-9.

[2] Gao, Y.; Shi, Z.-M.; Long, Z.; Wu, P.; Zheng, C.-B.; Hou, X.-D. Microchem. J. 2012, 103, 1.

[3] Yang, M.; Li, J.-X.; Wang, J.-H. Talanta 2007, 72, 1710.

[4] Yu, Y.-L.; Jiang, Y.; Chen, M.-L.; Wang, J.-H. Trac-Trends Anal. Chem. 2011, 30, 1649.
[5] Erxleben, H.; Ruzicka, J. Anal. Chem. 2005, 77, 5124.

[6] Hu, J.; Li, W.; Zheng, C.-B.; Hou, X.-D. Appl. Spectrosc. Rev. 2011, $46,368$.

[7] Meyer, C.; Muller, S.; Gurevich, E. L.; Franzke, J. Analyst 2011, $136,2427$.

[8] Zhu, Z.-L.; Zhang, S.-C.; Lv, Y.; Zhang, X.-R. Anal. Chem. 2006 78,865 .

[9] Zhu, Z.-L.; Zhang, S.-C.; Xue, J.-H.; Zhang, X.-R. Spectrosc. Acta Part B-Atom. Spectr. 2006, 61, 916.

[10] Pascoa, R.; Toth, I. V.; Rangel, A. Talanta 2011, 84, 1267

[11] Avivar, J.; Ferrer, L.; Casas, M.; Cerda, V. Anal. Bioanal. Chem. 2011, 400, 3585

[12] Tao, S.-Q.; Gong, S.-F.; Xu, L.; Fanguy, J. C. Analyst 2004, 129, 342.

[13] Yu, Y.-L.; Du, Z.; Chen, M.-L.; Wang, J.-H. Angew. Chem., Int. Ed. 2008, 47, 7909 . 